\title{
Kadi II: Europas Freiheitsrechte werden in Luxemburg verteidigt
}

VB verfassungsblog.de/kadi-ii-europas-freiheitsrechte-werden-in-luxemburg-verteidigt/

Matthias Kottmann Mo 22 Jul 2013

Der sogenannte Kampf gegen den Terrorismus wird vorwiegend mit Informationen geführt. Mächtig ist, wer sie besitzt und über ihre Verwendung bestimmt. Die Europäische Union hat sich in diesem Kampf mit der Rolle des Erfüllungsgehilfen begnügt. Sie hat Sanktionen gegen einen Mann erlassen, auf Grundlage zweifelhafter amerikanischer Geheimdienstinformationen, in die sie selbst keinen Einblick hatte. Mit dem Kadi II-Urteil verwirft nun der EuGH diese Rollenverteilung endgültig als Verstoß gegen europäische Grundrechte. Die Entscheidung, der zwölf Jahre rechtlicher Auseinandersetzung in unterschiedlichen Foren vorausgingen, zeichnet ein grelles Bild der von vernetzten Exekutiven dominierten „Neuen Weltordnung“. Sie zeigt indes auch einen Gerichtshof, der - vor der öffentlichen Aufmerksamkeit versteckt im Märchenland Luxemburg und nach Meinung mancher angeblich ohne die Legitimität und den Willen zu einer ernsthaften Grundrechtsrechtsprechung - zum bedeutenden Akteur in der Auseinandersetzung zwischen Freiheit und Sicherheit gereift ist.

Dreh- und Angelpunkt der Entscheidung ist ein Aspekt des Kadi I-Urteils, der gegenüber dessen viel diskutierten Ausführungen zum Verhältnis von Völker- und Europarecht weniger beachtet wurde: Das Ringen um Informationen. Die Unionsgerichte, so lautete es seit 2008 aus Luxemburg, müssten eine „grundsätzlich umfassende Kontrolle“ der europäischen Antiterrormaßnahmen vornehmen, auch wenn diese auf Resolutionen des UN-Sicherheitsrates beruhten. Dies umfasse insbesondere die „Nachprüfung [...] der angeführten Beweise und Informationen“, die gegen eine des Terrorismus verdächtigte Person vorlägen. Da die Unionsorgane die verlangten Belege nicht lieferten, könne die Judikative ihrer Kontrollaufgabe nicht nachkommen. Folglich sei das Grundrecht auf effektiven Rechtsschutz verletzt.

Diese Argumentation stößt sich allerdings mit den bis heute wenig veränderten Usancen der Terrorismusbekämpfung nach 9/11: Internationale Gremien beschließen Sanktionen gegen bestimmte Personen, die anschließend auf nationaler oder europäischer Ebene umzusetzen sind. Keine dieser Institutionen hat jedoch umfassenden Zugang zum gegen diese Personen vorliegenden Beweismaterial. Dieses verbleibt weitestgehend unter Verschluss desjenigen Staates, der es gesammelt hat. Selbst wenn also Rat, Kommission und die Mitgliedstaaten guten Willens wären - sie könnten dem EuGH nicht mehr vorlegen, als (in der Praxis offenbar meist, welch' Überraschung, amerikanische) Geheimdienstkreise innen zu überlassen geneigt sind.

Und während nun die Europäer - getrieben vom EuGH und seinen Nachahmern - im UN-Sicherheitsrat mit mittlerem Erfolg auf rechtsstaatliche Reformen drangen, zogen weitere Terrorverdächtige nach Luxemburg, und bekamen nach der soeben beschriebenen Logik allein aufgrund der allgegenwärtigen Geheimhaltungswut Recht. Herr Kadi war inzwischen mit neuen Sanktionen belegt worden und hatte diese erfolgreich vor dem EuG angefochten. In Gefahr, als unzuverlässige Terrorbekämpfer zu gelten, wandten sich der Rat, die Kommission und 13 Mitgliedstaaten erneut an den EuGH. Ihre Forderung: nicht weniger als eine umfassende Korrektur der bisherigen Rechtsprechung. Da Herr Kadi zwischenzeitlich - ohne veröffentlichte Begründung - auf UN-Ebene von der Liste der Terrorverdächtigen gestrichen worden war, ging es nur mehr ums Prinzip. Die juristischen Argumente waren ohnehin im Wesentlichen ausgefochten, es blieb die Frage, ob der EuGH angesichts dieser Kulisse einknicken würde.

\section{Machtprobe bestanden}

Der EuGH besteht die Machtprobe. Fast erscheint es als vorausschauende Planung, dass er die zentralen Grundsätze und Argumente seit Kadi-l bei jeder Gelegenheit wiederholt hat, um nun darauf zurückgreifen zu 
können. Kühl stellt er demnach fest, es sei

keine Entwicklung eingetreten, die es rechtfertigen könnte, diese Entscheidung in Frage zu stellen.

Auch in der Kontrolldichte will der Gerichtshof keine Abstriche machen. Insoweit hatte noch Generalanwalt Yves Bot an der Grenze rechtswissenschaftlicher Seriosität argumentiert, dass die Unionsgerichte - von Fällen offensichtlicher Personenverwechslung abgesehen - nicht überprüfen dürften, ob mit Sanktionen belegte Personen tatsächlich in terroristische Aktivitäten verstrickt seien. Anders der EuGH: Er sieht sich durch Art. 47 der Grundrechtecharta in der Pflicht, die Tatsachengrundlage von Sanktionsmaßnahmen zu überprüfen,

so dass sich die gerichtliche Kontrolle nicht auf die Beurteilung der abstrakten Wahrscheinlichkeit der angeführten Gründe beschränkt, sondern auf die Frage erstreckt, ob diese Gründe [...] erwiesen sind.

Bereits im Verwaltungsverfahren habe deshalb die zuständige Unionsbehörde erforderlichenfalls den UNSicherheitsrat und die betreffenden Drittstaaten um Übersendung der einschlägigen Informationen zu bitten.

\section{Zwei Innovationen}

Nun ändern diese Ausführungen nichts an dem Umstand, dass die betreffenden Drittstaaten nach wie vor wenig Neigung verspüren, geheimdienstliche Informationen an die Kommission oder gar den EuGH zu übermitteln. Dessen eingedenk wartet das Urteil mit zwei Innovationen auf: Erstens führt es richterrechtlich die nach der bisherigen Verfahrensordnung so nicht bestehende Möglichkeit eines in-camera-Verfahrens ein. Der EuGH anerkennt, dass es aus Sicherheitsgründen unabdingbar sein kann, bestimmte Informationen und deren Herkunft vor einem mutmaßlichen Unterstützer des Terrorismus geheim zu halten - wohlgemerkt aber nicht vor den Unionsgerichten. Sollte ein solcher Fall gegeben sein, was wiederum richterlicher Kontrolle unterliegt, so sind die vertraulichen Beweise allein vom Gericht einzusehen. Als Eingriff in das Recht des Klägers auf ein kontradiktorisches Verfahren ist dies freilich auf das Allernötigste zu beschränken und durch bestimmte Vorkehrungen weitestmöglich zu kompensieren.

Zweitens vollzieht der Gerichtshof eine punktuelle und nur schlecht kaschierte Kehrtwende: Falls Rat und Kommission trotz allem nicht in der Lage sein sollten, sämtliche Beweise für die erhobenen Vorwürfe zu liefern, so folge daraus nicht automatisch eine Verletzung des Grundrechts auf effektiven Rechtsschutz. So habe er, der Gerichtshof, das im Kadi-I-Urteil gar nicht gesagt. Es sei vielmehr schlicht so, dass die Unionsgerichte dann die unbewiesenen Vorwürfe ignorieren und folglich entscheiden müssten, ob die Verhängung von Sanktionen allein auf Grundlage der tatsächlich vorgelegten Beweise gerechtfertigt sei. Gewiss weicht der EuGH damit seine bislang kompromisslose Linie ein Stück weit auf. In der Sache bedeutet es freilich kein allzu großes Zugeständnis: Legen Rat und Kommission zu wenig Informationen vor, so folgt daraus zwar kein automatischer Verstoß gegen das verfahrensrechtliche Grundrecht auf effektiven Rechtsschutz, es droht aber nun regelmäßig das Rechtswidrigkeitsurteil aus materiellen Gründen.

Dass der EuGH den Fokus von der ehedem bevorzugten Kontrolle von Verfahrensrechten auf eine materielle Überprüfung verschiebt, ist bemerkenswert. Es zeigt meines Erachtens, dass man in Luxemburg zunehmend gewillt ist, sich mit schwierigen Beweis-, Abwägungs- und Verhältnismäßigkeitsfragen zu beschäftigen. Etwas anderes dürfte den Richtern auch nicht übrig bleiben, denn im Verfahren zur Vorratsdatenspeicherung steht schon bald die nächste grundlegende Entscheidung zwischen Freiheit und Sicherheit an. Es scheint also, als müsste man sich daran gewöhnen, dass künftig neben Karlsruhe und Straßburg ein dritter Akteur auf der Grundrechtsbühne mitspielt. 
Ob dessen Eigenheiten mag noch mancher in der deutschen Rechtsdogmatik Sozialisierter den einen oder anderen Kulturschock erleben. In der Sache macht er seine Arbeit aber gar nicht so schlecht.

LICENSED UNDER CC BY NC ND

SUGGESTED CITATION Kottmann, Matthias: Kadi II: Europas Freiheitsrechte werden in Luxemburg verteidigt, VerfBlog, 2013/7/22, http://verfassungsblog.de/kadi-ii-europas-freiheitsrechte-werden-in-luxemburg-verteidigt/. 